\title{
The Comprehensive Performance Evaluation of AODV and OSPF in WiMAX
}

\author{
Renuka Sharma \\ Department of CSE \\ NIET, Greater Noida
}

\author{
Pankaj Kumar \\ Department of CSE \\ NIET, Greater Noida
}

\author{
Devottam Gaurav \\ Department of CSE \\ NIET, Greater Noida
}

\begin{abstract}
Technologies based on IEEE 802.16 known as Worldwide Inter-Operability for Microwave Access (WiMAX) promises to deliver high data rate over long distance and multimedia services. WiMAX is a nascent in the field of communication operates in MAC and physical layer. It is mainly intended to overcome the drawback of the previous version. It is hottest in broadband wireless communication systems are demand for high data rates over a long transmission range and a minimum end to end delay. The high point of WIMAX is its performance metrics. In this paper, we discuss about the study and simulation of two routing protocol (i.e. ADOV and OSFP) for WIMAX mesh network based on different parameters (i.e. throughput, end to end delay, packet delivery ratio, routing-load) using NS-2.
\end{abstract}

\section{Keywords}

WIMAX, ADOV, OSPF, NS2.34

\section{INTRODUCTION}

Today's broadband Internet connections are limited to wiredinfrastructure using digital subscriber line (DSL), T1 or cablemodem based connection. However, these wired infrastructures are considerably more costly and timeconsuming to deploy than a wireless connection. Whenever, in undeveloped areas and developing countries, provide are unwilling to install then required equipment including an optical fibre or copper wire, etc. other for broadband services expecting the low profit. Broadband Wireless Access (BWA) has emerged as a promising solution for large distance access technology to provide very high-speed connections. IEEE 802.16 standard for Broadband Wireless Access and its linked industry consortium, WiMAX Forum promise to deliver high data rate over the long distance to a large number of customers, where broadband is unreachable. This is the first industry-wide standard that can be used for fixed wireless access (IEEE 802.16d) with substantially higher bandwidth than most cellular networks [11]. Development of this standard provides the low-cost equipment, ensure interoperability, and reduce investment risk for operators. WiMAX is one of the nascent and hottest broadband wireless technologies around today. WiMAX systems are expected to deliver broadband access services to residential and enterprise users in an economical way. WiMAX would operate or work similar to $\mathrm{Wi}-\mathrm{Fi}$, [7] but at higher speeds over larger distances and for a greater number of customers. WiMAX can provide service even in areas that are most difficult for wired infrastructure to reach and the capability to overcome the physical limitations of traditional wired infrastructure. The Wireless system is considered to be a perfect and attractive solution to provide high data rates over large distance communications, particularly for mobile users. The IEEE 802.16 standards are also known as WiMAX standards, are intended to offer wireless broadband access for the long range propagation. WiMAX is based on Wireless Metropolitan Area Network (WMAN) which provides very high data throughput over long distance (20 or 30 miles) in Non-Line of Site (NLOS) propagation [12]. This technology aims to provide broadband wireless access as well as internet access. The IEEE 802.16 standards have divided the WiMAX system into two groups [12], Fixed WiMAX (IEEE 802.16d-2004), Mobile WiMAX (IEEE 802.16e-2005). It is defined as IEEE 802.16d -2004 standards, Fixed WiMAX system based on the Wireless MAN-OFDM physical layer specifications with 256 carriers. Fixed WiMAX delivers point to multipoint (P2M) broadband wireless services to our homes and offices. WiMAX forum promises to offer high data rate over longdistance to a large number of users where broadband service is unreachable. The Forum defines WiMAX as "it is based on IEEE $802.16 \mathrm{~d}$ and authorises the delivery of last mile wireless broadband access as an alternative to cable and DSL". An air interface based on orthogonal frequency division multiplexing (OFDM) is used by it, which is very secure against multi-path transmission a frequency selective fading. An adaptive modulation technique is used to increase performance when the link characteristics vary. In fixed WiMAX system used Frequency Division Duplexing (FDD), where the Base Stations (BSs) and the user terminals transmit in different frequency bands. The IEEE.802.16 standard defines MAC layer, which is connection oriented and uses a Time Division Multiplexing (TDM) for the downlink (DL) and a Time Division Multiple Access (TDMA) schemes for the uplink (UL). This display the Point to Multipoint (PMP) architecture [6].Mobile WiMAX supports fixed, nomadic, mobile, portable application and it Operates in a frequency band of $2 \mathrm{GHz}$ to $6 \mathrm{GHz}$ and the Transmission range up to $75 \mathrm{Mbps}$ for the distance 10 miles $(15 \mathrm{Kms})$ with Multicarrier signal (OFDM) is used. It is defined as IEEE 802.16e-2005 standards, offers scalability in both radio access technology and network architecture, so it provides flexibility in network deployment and service offerings. Mobile WiMAX adds significant improvements:

- It improves non-line of sight coverage by utilizing advanced antenna diversity schemes and hybrid automatic repeat request (HARQ).

- It selects dense sub-channelization, so it was enhancing system gain and improving indoor penetration. It uses multiple input multiple output (MIMO) technologies and adaptive antenna system (AAS) to improve service offering coverage. It defined a downlink sub-channelization scheme, enabling better coverage and capacity trade-off.

WiMAX networks consist of a Base Station (BS) and some Subscriber Stations (SS). In Mobile WiMAX network, Base Station which is fixed is connected to a public network and can handle multiple sectors simultaneously and Subscriber Station is mobile. There are different wireless routing protocols are designed to provide communication facility in the wireless environment, such as AODV, OLSR, DSDV, ZRP, LAR, RIP, DSR and IGP etc. But routing in wireless 
network is very challenges task due to high mobility and frequently changes of nodes. This paper presented an performance analysis of reactive routing Protocols (AODV) and proactive protocols (OSPF) in WIMAX environment based on different parameters (i.e. throughput, end to end delay, packet delivery ratio, routing-load) using different scenarios.

\section{RELATED WORK}

In 2016, A.B Ali, et. al. [17], discussed in this an author find out the best protocol combination for any complex scenario to achieve fast and reliable communication. In this they used Hot Standby Routing Protocol (HSRP) and Gateway Load Balancing Protocol (GLBP) is also simulated to analyze the load balancing and redundancy parameter for Border Gateway Protocol (BGP). Based on the simulation results it can be observed that EIGRP and OSPF are the best combination of protocols for a given network within 1000 hosts. However, a combination EIGRP and RIPv2 would be better suited for a smaller network because of the absence of segmented areas. In this IS-IS has been also known as the best protocol for ISP's and really large enterprises because of its scalability, fast convergence and added the advantage of not needing IP connectivity to be able to communicate with neighbors. The results also conclude that it communicates well with OSPF, due to their similarities. So the author discussed that, the combination of the two protocols would be better than configuring only 1 of them for any given scenario with complex parameters.

In 2016, T. Sharma et. al. [19], discussed an analysis of ADOV, DSR and DSDV protocol for WiMAX mesh network based on average end to end delay, packet delivery ratio, throughput and normalized routing load has been done. Successfully results found that AODV protocol outperform the DSR and DSDV.

In 2015, P. Ashar [18], discussed in this author summarizes the features of some of the most significant protocols like RIP, OSPF, IS-IS, IGRP, EIGRP and BGP, while comparing their individual functioning.

In 2015, F. Anwar et. al. [17], discussed this paper provide a focus upon those routing protocols especially designed for wireless networks. In which author compare the performance of four wireless routing protocols (AODV, DSR, OLSR and ZRP) for Mobile WiMAX environment under the assumption that each of the mobile station has routing capabilities within its own network. From simulation, they found that ZRP and AODV protocols outperform DSR and OLSR.

In 2014, G. Kaur et. al. [16], discussed an analysis of those routing protocols especially designed for wireless networks. A study and comparison on the performance of reactive protocol (AODV) and proactive protocols (OLSR, DSDV) for Mobile WiMAX environment is done under varying mobility conditions. The performance matrix includes Packet Delivery fraction (PDF), Throughput, End to End Delay, and routing load were identified. In this author used NS2 simulator for the compare the performance analysis. From the results they found that AODV protocol out-perform the DSR and DSDV.

In 2012, K. Khandakar[15], discussed in this author does Comparison of DSR, AODV and DSDV based on performance metrics Packet Delivery Fraction, End to end delay and Normalized Routing load while changing the number of nodes, speed and Pause time. AODV has a stable End to End Delay despite mobility as it has the feature of OnDemand Routing protocol and also maintains a Routing table.
DSDV has a higher Packet delivery fraction than the other two routing protocols in mobility as it is a Table Driven protocol and is more reliable.DSR has the highest End to End Delay and Routing load increases the bandwidth and consuming the battery life. Based on the simulation scenario, parameter, assumption, the results of AODV could be considered as an efficient faster routing protocol than DSR and DSDV but has a higher Routing load comparatively than DSDV.

In 2008, K. Etemad et. al. [14], discussed this paper provides a high-level overview of mobile WiMAX technology and its evolution from both radio and network perspectives. The technology utilizes advanced PHY and MAC techniques in radio to provide high band efficiency and quality of service (QoS) control as well as IP-based flat network architecture supporting multivendor plug and play deployments. Mobile WiMAX has defined the technology evolution roadmap for the next few years, which includes, but goes beyond, further improvements in system efficiency and user experience.

\section{WIRELESS ROUTING PROTOCOLS 3.1 Mobile Ad Hoc Network (MANET)}

MANET itself stand for Mobile Ad Hoc Networks, in which each node act as router and it can move freely anywhere in the network. In other words, Mobile Ad Hoc Networks is selforganizing network, in which each node communicates with each other without relying on existing infrastructure and wirelessly [23].The feature of self-organizing and self administration make it applicable for various applications, such as military operation, wireless mesh network and wireless sensor network etc. al. Mobile Ad Hoc Networks can allow multihop to the destination and support for mobility with various features (robustness, rapid deployment, flexibility, etc.). Mobile Ad Hoc Networks can be classified into Table-Driven and On-Demand Routing protocol where Table Driven protocols are proactive and maintain a routing table or it is static in nature and On-Demand are active and do not maintain a routing table or dynamic in nature [15], and a combination of reactive and proactive are called hybrid routing.

Reactive Protocols: it is also known as on-demand routing protocols. These protocols initialize the routing process whenever a node requires otherwise the network is going to sleep. These are considered efficient, where the route discovery is required to be less frequent. This makes them more suitable to the network with less traffic and low mobility [16]. Examples of reactive protocols are Ad-Hoc on Demand Distance Vector Routing Protocol (AODV), and Dynamic Source Routing (DSR).

Proactive Protocols: The table driven approach is similar to the connectionless approach of forwarding data packets, with no regard to when and how frequently such routes are desired. It relies on an underlying routing table update mechanism that tables are updated regularly in order to maintain a up-to-date routing information from each node to every other node. Here, a route to every other node in ad-hoc network is always available, regardless of whether or not it is needed [23]. These protocols are used where the route requests are frequent [16]. Examples of such protocol are DSDV (Destination Sequence Distance Vector Routing Protocol), OSPF (Open Shortest Path First), etc.

Hybrid Routing Protocols: These protocols combine the advantages of the two routing protocols in order to gain higher efficiency. In these a network is portioned in to the zones, in 
which if the routing is to be carried out within the zone than table driven routing is used else on demand routing is used [23]. Example of such Protocol is Dynamic MANETs Ondemand Routing.

Here we are discussing those routing protocols used in the simulation process.

\subsection{Ad hoc On-demand Distance Vector Routing (AODV)}

AODV is a reactive routing protocol which is combination of DSDV and DSR. Route is calculated on demand, just as it is in DSR via route discovery process [15]. Even though ADOV is reactive routing protocol still it maintain a table. ADOV offers quick alteration to dynamic link condition, low memory overhead, low network consumption, low dispensation, and unicast route purpose to destination within the ad hoc network. In ADOV distance and path calculated on demand i.e. when the request is sent from the source node to a subsequent node. It uses a destination sequence number (DSN) to ensure loop free route all the times, avoiding trouble [19].

The ADOV protocol divided into two major phases:[19]

- Route discovery

- Route maintenance

\subsubsection{Route discovery}

When a node wants to send a packet to some destination node and does not find a valid route in its routing table, it initiates a route discovery process. Source node broadcast a route request (RREQ) packet to its neighbors [17], an intermediate node receives a RREQ broadcast massage it copies the address of a node from which it has received massage. It then appends its RREP massage to the broadcast massage this creating a reverse path. This path is used to unicast the massage back to back to the destination [19].

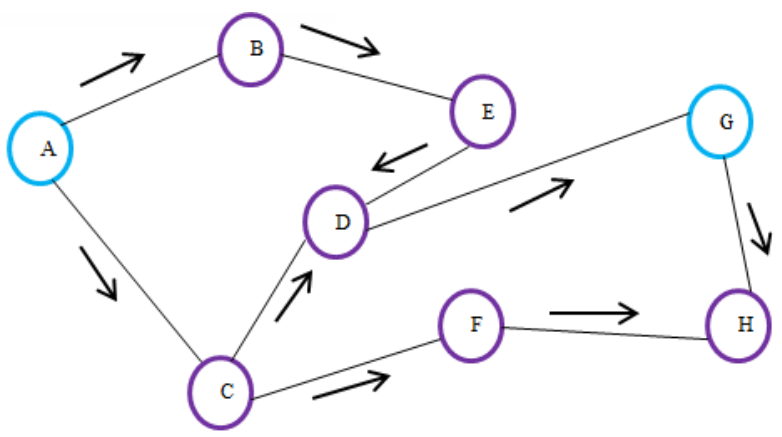

Figure 1: Propagation of route request throughout the network

\subsubsection{Route maintenance}

A node is connected to the route path till an active connection is needed however, in certain circumstances if the source node moves further away from its adjacent path node then it can easily reinstate a connection back to the adjacent node and restart communication. If an intermediate node disconnects for this purpose, the effected node broadcast a RERR message to its upstream neighbor [19].

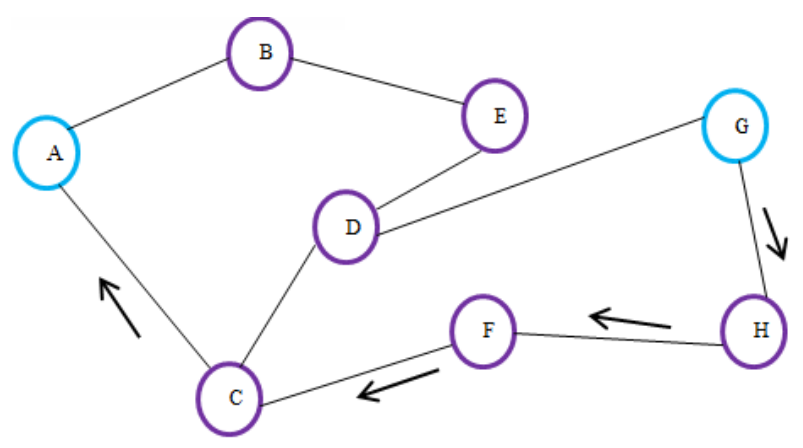

Figure 2: Reply of RREP towards the network

Objective of ADOV routing protocols: [25]

- $\quad$ AODV diminish the control overhead by decreasing the number of transmits using a pure on- demand route acquisition method. ADOV uses only symmetric link between adjacent nodes.

- Unicast, Broadcast, and Multicast communication.

- On-demand route establishment with small delay.

- To differentiate between local connectivity management (neighborhood discovery) and general topology protection [23].

- To transmit discovery packets only when it is necessary.

- To publicize about changes in local connectivity to those adjacent mobiles node which are need of such information

\section{Limitation of $A D O V$}

- Intermediate nodes can lead to inconsistent routes if the source sequence number is very old and the intermediate nodes have a higher but not the latest destination sequence number, so it creates problem of stale entries [25].

- High latency time in route finding.

- Excessive flooding can lead to network congestion.

- Multiple Route Reply packets in response to a single Route Request packet can lead to heavy control overhead.

- Unnecessary bandwidth consumption due to periodic beaconing (A continuous signaling of error conditions)

\subsection{Link State Routing Protocol (Open Shortest Path Fast)}

OSPF is a link state routing protocol developed by Interior Gateway Protocol (IGP) working group of the Internet Engineering Task Force (IETF) for Internet Protocol (IP) network [18]. As an IGP, OSPF distributes routing information between routers belonging to a single autonomous system (AS). An Autonomous System (AS) is a collection of routers under a common administration is known as a routing domain. OSPF was defining as an improvement to RIP, with faster convergence and more configurable parameters [20]. Open Shortest Path Vector (OSPF) is a link state dynamic routing protocol, which maintains the routing table for all connections in the network [18]. It sends out hello packets, link state requests, updates and database descriptions, 
and applies the Dijkstra's algorithm to determine the shortest path to the destination [20]. Cost is a metrics used to describe the relative efficiency of various routes to the destination. OSPF runs on top of IP, i.e., an OSPF packet is transmitted with IP data packet header.

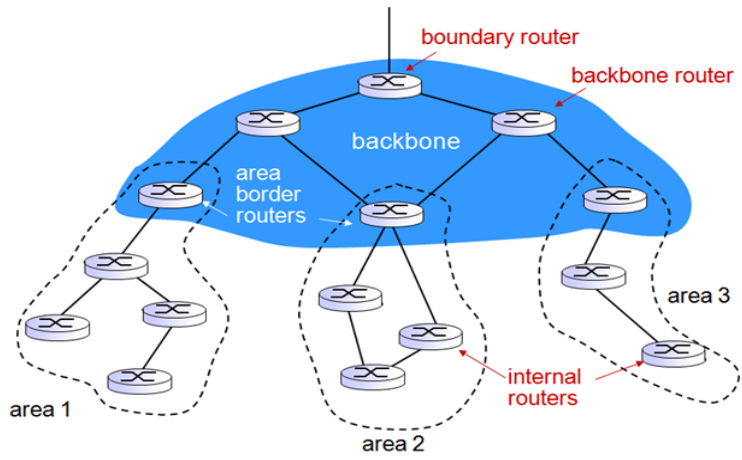

Figure 3: Hierarchical OSPF Structures [7]

In OSPF large network divided into smaller internetworks called Areas. An area is a logical collection of OSPF networks, routers, and links that have the same area identification. A router within a same area maintains a topological database for the area to which it belongs. The router does not contain information about network topology out of its area, so reducing the size of its database. OSPF is a good example of fast convergence [25]. A network of few routers can converge in a matter of seconds. It is one of the main design goals and an important performance indicator for routing protocols to all areas must be connected to Area0 with ABR Area Border Router, Maintaining separate link-state databases for each area and connects different areas with the backbone area i.e. Area 0. All the routers within same area have same topology table. The goal of design is to localize the updates within area. Autonomous System Border Router (ASBR) is used to import non-OSPF network information to the OSPF network. It connects different autonomous systems. In figure 3: define the area design of OSPF routing protocol. The OSPF divides the network into small areas with fixed no. of router to minimize the routing update traffic and it limit the scope of route information distribution. The link-state database (LSDB) is used to calculate the best paths through the network of routers [25]. In this router used the LSDB information to find shortest path through the network.

\section{Advantages of OSPF}

- OSPF is not a proprietary protocol.

- Low bandwidth utilization, Multiple routes are supported.

- OSPF always determine the loop free routes.

- If any changes occur in the network it updates fast.

- It is suitable for large network.

Challenges related to $O S P F$

- It is difficult to configure.

- It has Link state scaling problem.

- It requires more memory for configuration.

\section{PERFORMANCE METRICES}

The performance metrics chosen for performance evolution of WiMAX are packet delivery ratio, throughput, normalized routing load, and end to end delay:

\subsection{Packet delivery fractions (PDF)}

It is define as the ratio of number of packets received by the receiver to the number of packets send by the source. In other words it is fraction of packet sent by the source that is received by receivers [23]. The ratio represents the effectiveness of the routing protocol. It specifies the packet loss rate, which limits the maximum throughput of the network [18]

$$
P=\frac{1}{c} \sum_{f=1}^{e} \frac{R_{f}}{N_{f}}
$$

Where $\mathrm{c}$ is total connections to destination, $\mathrm{f}^{\text {th }}$ connection is index to connection to it. $R_{f}$ is no. of received packets by $\mathrm{f}^{\text {th }}$ connection. $N_{f}$ is no. of packets sent over to the destination through $\mathrm{f}^{\text {th }}$ connection. Higher PDF value means better performance of the protocol [17].

\subsection{Average End-to-End Delay (EED)}

It is the average time when a packet is sent from the source node and is successfully received by the receiver's node. It includes delays as delay for route discovery, propagation time, data transfer time, and intermediate queuing delays [20].it is measured in millisecond (ms). When particular packet " $\mathrm{i}$ " is sent at $s_{i}$ time and received at $r_{i}$ time delayed due to all these delays. Average for all the packets sent is given by [17]:

$$
D=\frac{1}{N} \sum_{i=1}^{s} r_{i}-s_{i}
$$

\subsection{Throughput}

The throughput can be refers as percentage of the packets received by the destination among the packets sent by the source [20]. It is measured in kilo byte per second (Kbps). Some factors affect the throughput as, topology changes in the network, unreliable communication between nodes, limited bandwidth available and limited energy [24]. Throughput can be represented in equation below [17].

$$
\text { Throughput }=\frac{\text { No. of bytes receoved } \times 8 \mathrm{KBPS}}{\text { Throughput simulation time } \times 1000}
$$

\subsection{Normalized Routing Load (NRL)}

It is defined as the ratio of number of routing packets transmitted per data packets received [23].

$$
N R L=\frac{\text { No. of routing packet } \times 100}{\text { No. of packet received }}
$$

\section{NS-2 SIMULATOR}

There are many simulators such as Network Simulator 2 (NS2), OPNET Modeler, GloMoSim, OMNeT++ and many others. In this paper we have chosen a Network Simulation Tool NS-2 (version 2.34). NS2 which is an object-oriented, discrete event driven network simulator developed at UC Berkeley that focuses on the simulation of IP networks on the packet level. The network simulator version 2.34 is a package of tools that simulates behavior of networks. It can simulate both fixed and wireless network. But NS-2 (versionNs-2.34) is a mostly used in wireless network simulation tool for this purpose. In which two language are used, $\mathrm{C}++$ and Tool Command Language (TCL). It uses TCL/OTCL (Tool Command Language/ Object Oriented TCL) as a command \& configuration interface. Basically in NS-2.34 C++ is its backend language and TCL is its scripting and frontend language. NS-2.34 includes a tool for viewing the simulation 
results, called Network Animator (NAM). It uses three types of files namely Tool Command Language file (.tcl), Trace file (.tr) and Network Animator file (.nam). Tool command language file (.tcl) has subsets of commands which are written into it for simulation. While simulator runs on tcl, simulation trace file (.tr) and animation file (.nam) are created during the session. Trace file (.tr) is used to trace the whole process and Network Animator file (.nam) is used to visualize the behavior of network protocols and traffic the model.

\subsection{Simulator Parameters}

The simulation is carried out with the help of NS-2 (V-2.34) network simulator. The NS-2 provides good implementations of the different network protocol. There is no. of simulation parameters show in table 1.

All the simulation work is carried out in wireless network simulator NS-2(V-2.34) have been designed within a terrain area of $748 \mathrm{~m} \times 473 \mathrm{~m}$. Mobility model is used Random Way Point (RWP). In this model, a mobile node is placed randomly at any location in the simulation area. For simulation speed of node changes from $10 \mathrm{~m} / \mathrm{s}$ to $60 \mathrm{~m} / \mathrm{s}$. packet size of each datagram is 1000 byte. Each CBR source sends packets at the rate of $0.01 \mathrm{MB}$. The traffic pattern was generated using CBR as the data source and UDP protocol is used for transporting the data and the packet size is of 1000 bytes. The simulations are done for different scenarios by varying the number of nodes, speed of the nodes.

Table 1: Simulation Parameters in NS2

\begin{tabular}{|l|l|}
\hline SIMULATOR PARAMETERS & \multicolumn{1}{|c|}{ VALUE } \\
\hline Simulator & NS-2.34 \\
\hline Area & $748 \mathrm{~m} * 473 \mathrm{~m}$ \\
\hline Routing protocols & ADOV,OSPF \\
\hline Traffic & CBR(UDP) \\
\hline Simulation time & 200 seconds \\
\hline No. of nodes & $25,50,75, \ldots \ldots \ldots . .600,625$ \\
\hline Packet size & 1000 bytes \\
\hline Max. speed & $10 \mathrm{~m} / \mathrm{s}$ to $60 \mathrm{~m} / \mathrm{s}$ \\
\hline Max. connection & 5 \\
\hline Movement model & Random way point \\
\hline
\end{tabular}

\section{SIMULATION RESULTS}

The performance metrics chosen for performance evolution of WiMAX are packet delivery ratio, throughput, normalized routing load, and end to end delay:

\subsection{Simulation with varying no of nodes}

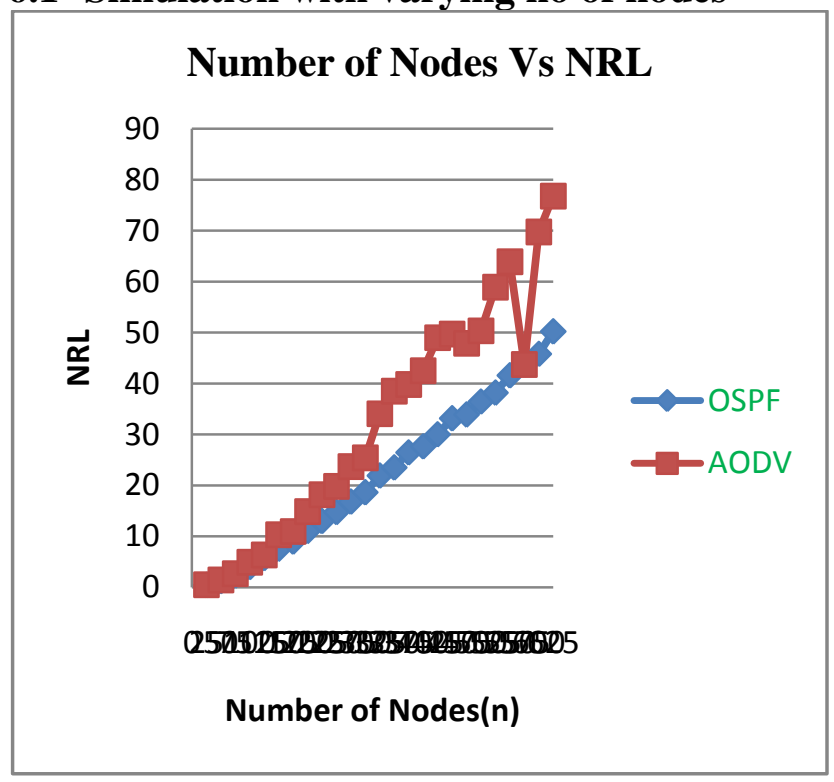

Figure 4: NRL vs. no. of node

Figure 4 shows number of nodes vs. normalized routing load with two routing protocol and different node densities $25,50,75, \ldots \ldots \ldots, 625$. This graph indicates the NRL for two routing protocol with respect to WIMAX network. From this graph we identified the OSPF perform well as compare to AODV. As the no. of node increase - increase in routing load in ADOV but at some point at it randomly fall due to less congestion in network, but overall performance of OSPF is best.

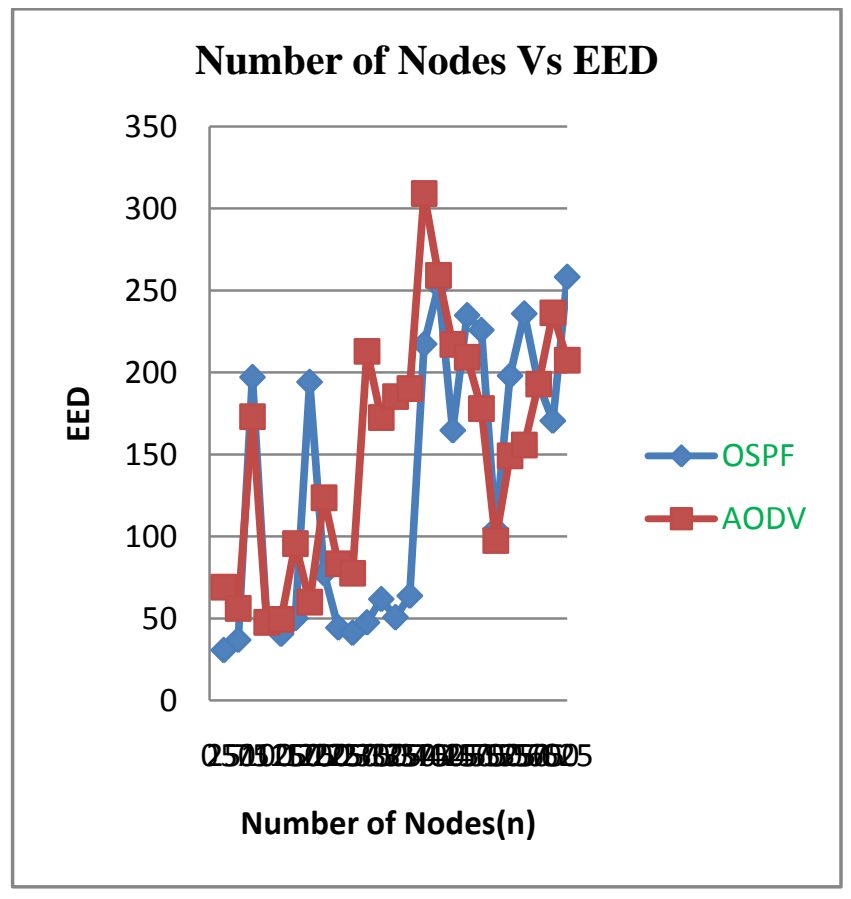

Figure 5: EED vs. no. of node

Figure 5 shows number of nodes vs. end to end delay with two routing protocol and different node densities $25,50,75, \ldots \ldots . .625$. This graph indicates the EED for two routing protocol with respect to WIMAX network. From this graph we identified the OSPF, ADOV perform avg. 


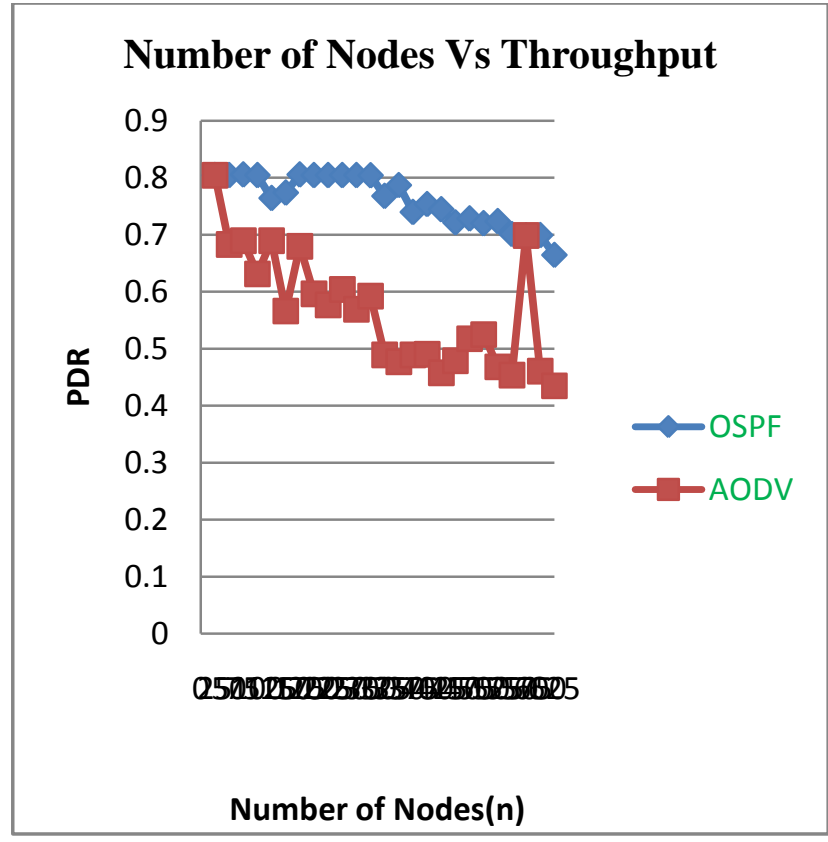

Figure 6: PDR vs. no. of node

Figure 6 shows number of nodes vs. throughput with two routing protocol and different node densities $25,50,75 \ldots \ldots \ldots$ 625. This graph indicates the throughput for two routing protocol with respect to WIMAX network. From this graph we identified the OSPF has a high PDR due to less traffic in network when compare to AODV.

\subsection{Simulation result with varying speed of node.}

Figure 7 shows speed of nodes vs. throughput with two routing protocol and different node densities 10, 20...60. This graph indicates the throughput for two routing protocol with respect to WIMAX network. The effect of speed variations for ADOV and OSPF has been examined. From this graph we identified the OSPF perform well (217 kbps to 227 $\mathrm{kbps}$ ) as speed $10 \mathrm{~m} / \mathrm{s}$ compare to AODV.

Figure 8 shows speed of nodes vs. normalized routing load with two routing protocol and different node densities 10, 20...60. This graph indicates the NRL for two routing protocol with respect to WIMAX network. The effect of speed variations for ADOV and OSPF has been examined. From this graph we identified the OSPF perform well as speed beyond $20 \mathrm{~m} / \mathrm{s}$ when compare to AODV. But ADOV also has avg. performance because increasing mobility of node increasing routing load.

Figure 9 shows speed of nodes vs. packet delivery ratio with two routing protocol and different node densities 10,20...60. This graph indicates the PDR for two routing protocol with respect to WIMAX network. The effect of speed variations for ADOV and OSPF has been examined. From this graph we identified the OSPF perform constant beyond $20 \mathrm{~m} / \mathrm{s}$ and AODV beyond $20 \mathrm{~m} / \mathrm{s}$ and before $40 \mathrm{~m} / \mathrm{s}$ started declining due to congestion in the network.

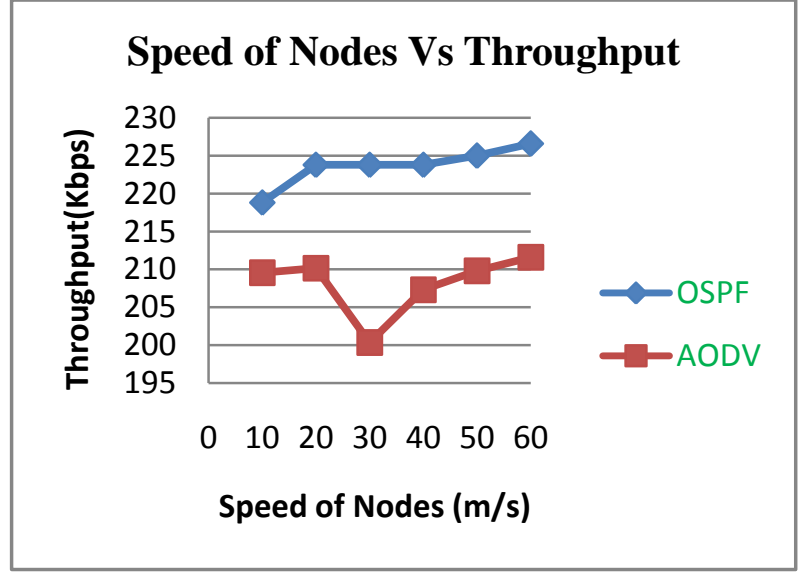

Figure 7: throughput vs. speed of nodes

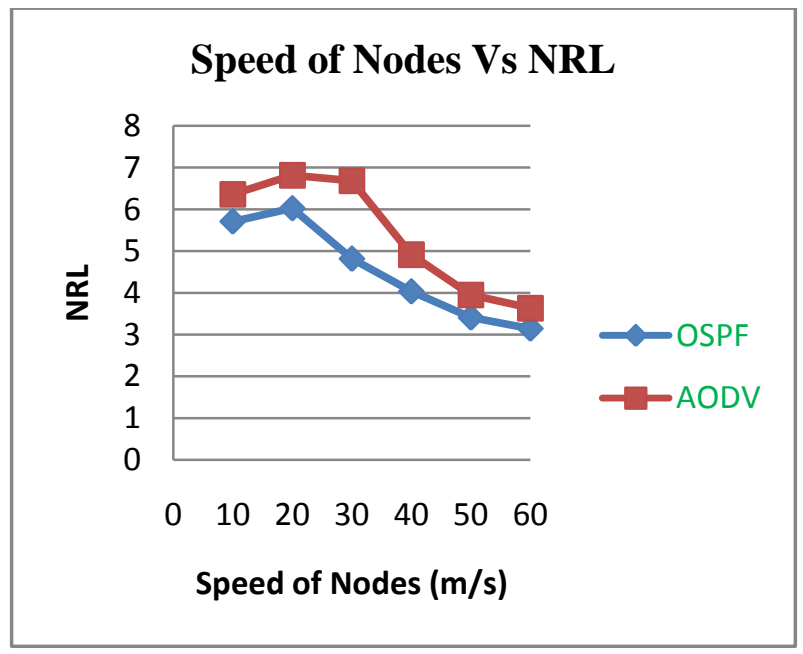

Figure 8: NRL vs. speed of nodes

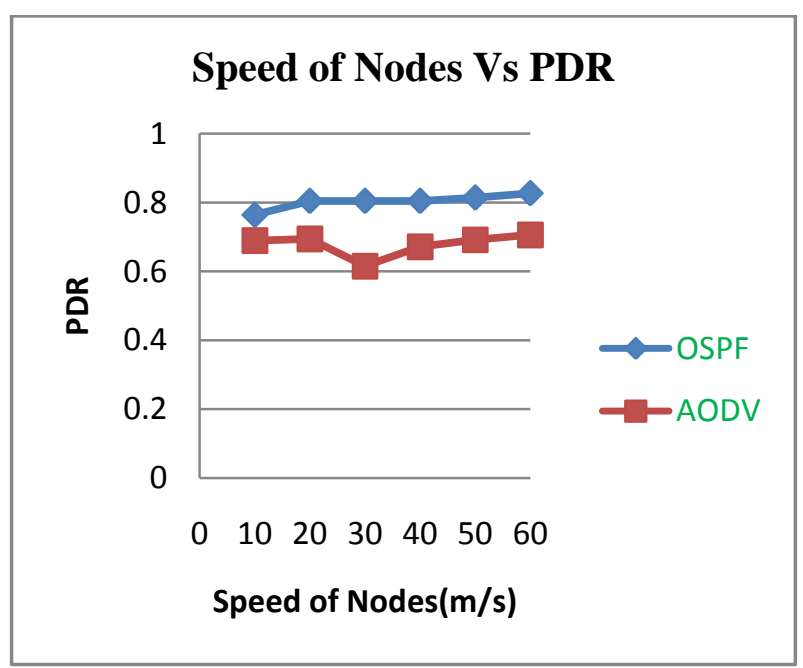

Figure 9: PDR vs. speed of nodes 


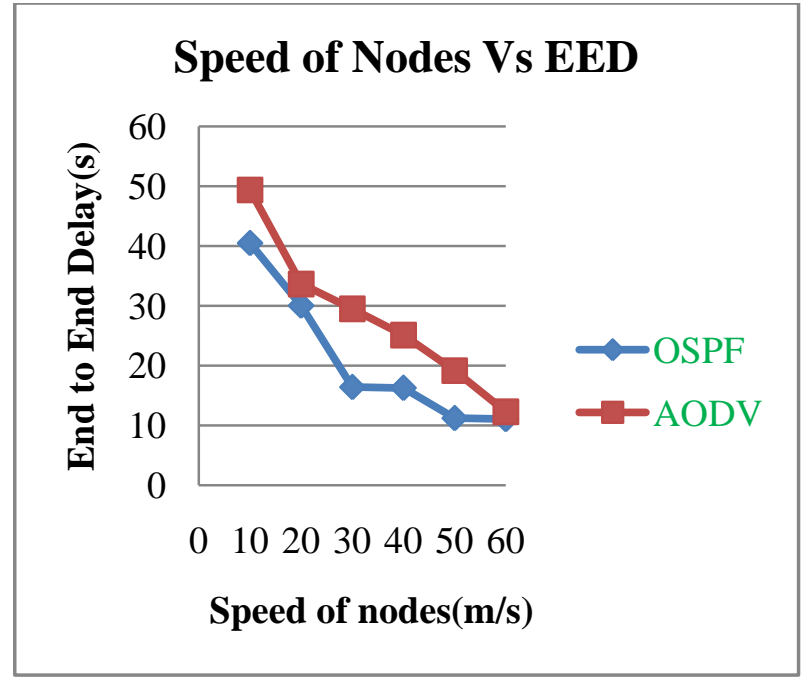

Figure 10: END vs. speed of nodes

Figure 10 shows speed of nodes vs. end to end delay with two routing protocol and different node densities $10,20, \ldots, 60$. This graph indicates the throughput for two routing protocol with respect to WIMAX network. The effect of speed variations for ADOV and OSPF has been examined. From this graph we identified the OSPF perform best as speed beyond $10 \mathrm{~m} / \mathrm{s}$ when compare to AODV.

\section{CONCLUSION}

WiMAX (Worldwide Inter-Operability for Microwave Access) promises to deliver high data rate over large distance and deliver multimedia services accesses anywhere anytime. Routing in WiMAX network is very challenging problem due to high mobility of node and the frequent topology changes. In this paper, we analyzed the performance of ADOV and OSPF routing protocols under different simulator parameters. From the obtained results, we analyzed OSPF (Open shortest path first) will give better output in all the four parameters against different scenarios (no. of node, speed of node). ADOV routing protocol also give better result but not best when compare to OSPF. Different routing protocol gives different result in various simulators. OSPF lives up to its protocol specifications because it performs well in a highly dense network even under varying load conditions. It gives a high throughput under most conditions, but at the cost of an increased overhead. The AODV is better for moderately dense networks where as the OSPF performs well in sparse networks. So in this paper OSPF is well suited for mobile WIMAX network.

\section{FUTURE SCOPE}

In our future work we focus on Other MANET routing protocols such as (DSR, DSDV, RIP... etc) could be taken with other parameters for further studies such as (number of hops per route, packet dropped, route error sent , retransmission attempts and buffer overflow

\section{REFERENCES}

[1] D. Pareit, Bart Lannoo, Jngrid Moerman, 2012, "The history of WIMAX: A complete survey in certification and standardization for IEEE 802.16 and WIMAX", IEEE communication surveys \& tutorials, Vol 14.

[2] M. Wysocki, T. Abolhasan, E. Dutkiewicz, 2004, "A review of routing protocols for mobile ad hoc networks," Elsevier, Amsterdam.
[3] S. Kumar Gupta, R.K. Saket, June 2011, "Performance Metric Comparison of AODV and DSDV Routing Protocols Using Ns-2," Volume 7.

[4] V. Kumar Jatav, Dr. Vrijendra singh, 2014, "Mobile wimax network security threat and solution: A survey," $5^{\text {th }}$ international conference on computer and communication technology.

[5] C. E. Perkins,E M Royer,Sameer R. Das, 2001, "Ad hocOn-Demand Distance Vector (AODV) Routing," InternetDraft, draft-ietf-manetaodv- 09.txt.

[6] Li. Chunlin, YaunPeiyan, February 2007, "Performance Evaluation and simulation of Routing Protocols in AdHoc Networks," Computer Communication.

[7] OverviewofWiMAX,http:www.tutorialspoint.com/WiM AX/index.html

[8] Navaneeth Y Krishnan, Chandan N Bhagwat, Aparajit Utpat, January 2013, "Performance Analysis of OSPF and EIGRP Routing Protocols for Greener Internetworking," IJCA Proceedings on International Conference in Distributed Computing and Internet Technology 2013, ICDCIT:1-5.

[9] OSPF routing protocol implementation: http://www.quvik.com/media/blog/ospf protocol explained

[10] M. Suresh, Mr G. Jabert, Mr C. Kamalanathan, Mr S. Kirubakaran, Dr S. Valarmathy, October 2013, "Improving the Performance of WIMAX Using Various Routing Protocols," International Journal of Advanced Research in Computer Science and Software Engineering, Volume 3, Issue 10.

[11] P. Dattaet et. al., 06-08 March, 2014, "Exploration and Comparison of Different 4G Technologies Implementations: A Survey," 2014 RAECS UIET Punjab University Chandigarh.

[12] D. Kene, D. Kulat, Jagdish, 08-10 DEC.2011, "Performance evaluation of IEEE 802.16e WIMAX physical layer", institute of technology, Nirma University, Ahmadabad 382481.

[13] M. Azizul Hasan, 2007, "Performance Analysis of WIMAX/IEEE 802.16 OFDM Physical Layer," Helsinki University of Technology, Finland.

[14] K. Etemad, Oct. 2008, Intel Corporation, "Over View of Mobile WIMAX Technology and Evolution" IEEE Communication Magazine.

[15] A. Khandaker, 2012, "Step by Step Comparison of DSDV, DSR and ADOV routing protocol," International conference on computer Engineering and Technology.

[16] G. Kaur1, Praveen Kumar, May 2014, "Performance Analysis of Reactive and Proactive Routing Protocols under Varying Mobility in WiMAX Environment," International Journal of Advanced Research in Computer and Communication Engineering Vol. 3, Issue 5.

[17] F.Anwar, Md. Saiful Azad, Md. Arafatur Rahman, Mohammad Uddin, 2008, "Performance Analysis of Ad hoc Routing Protocols in Mobile WiMAX Environment," International Journal of Computer Science. 
[18] P. Asher, 2015, "Comprehensive Analysis of Dynamic Routing Protocols in Computer Networks," (IJCSIT) International Journal of Computer Science and Information Technologies, Vol. 6 (5).

[19] T. Sharma, Parteek Handa, 2016, "Performance Analysis of ADOV, DSR and DSDV protocol for WiMAX mesh network," International Conference on Computing for Sustainable Global Development.

[20] A. Baba Ali, Mujahid Tabassum, Kuruvilla Mathew, March 2016, "A Comparative Study of IGP and EGP Routing Protocols, Performance Evaluation along Load Balancing and Redundancy across Different AS," International Multi Conference of Engineers and Computer Scientists, Vol. II, IMECS 2016, Hong Kong.

[21] J. Preet Kaur, Mandeep Kaur, July 2015, "Simulation Based Analysis of IEEE 802.16 Wimax Routing Protocols for High Load Applications," International Journal of Electronics \& Communication (IIJEC), Volume 3, Issue 7.

[22] D. Chauhana, Sanjay Sharma, 2014, "Performance Evaluation of Different Routing Protocols in IPv4 and
IPv6 Networks on the basis of Packet Sizes," International Conference on Information and Communication Technologies (ICICT)

[23] D. Gaurav, Charu Wahi, 2015, "Performance Evaluation of AODV with and without Black hole Attack in MANETs," International Journal on Recent and Innovation Trends in Computing and Communication ISSN: 2321-8169 Volume: 4 Issue: 6.

[24] S. Shrivastava, Ashok Verma, Ajay Lala, Ashish Chaurasia, May-June 2014, "A Study and Comparsion of Various Routing Protocol in WiMAX Network," International Journal of Emerging Trends \& Technology in Computer Science (IJETTCS), Volume 3, Issue 3, ISSN 2278-6856.

[25] Sukhmani, Vidhu Kiran, March - April 2015, "Review of Issues and Challenges in IEEE 802.16 WiMAX,' Advanced Journal of Computer Science and Engineering (AJCST), ISSN: 2393-8390 (O), Vol. 3, Issue 1.

[26] M. Azizul Hasan, 2007, "Performance Analysis of WiMAX/IEEE 802.16 OFDM Physical Layer," Department of electrical and communication engineering, communication laboratory, Helsinki University of Technology, Finland. 